\title{
Analysis of Accredited Gastroenterology Fellowship Internet-Available Content: Twenty-Nine Steps Toward a Better Program Website
}

\author{
Jack H. Ruddell ${ }^{1}$ - Adam E. M. Eltorai ${ }^{1}$ - Mena Bakhit ${ }^{2} \cdot$ Arman M. Lateef $^{1} \cdot$ Steven F. Moss $^{2}$
}

Published online: 13 March 2019

(c) Springer Science+Business Media, LLC, part of Springer Nature 2019

\begin{abstract}
Goal To assess publicly available content derived from official websites of accredited gastroenterology fellowship programs, specifically evaluating data pertinent to prospective applicants.

Background The Internet provides access to key information for applicants applying to gastroenterology fellowship, particularly as competition drives applicants to apply to a large number of programs. Thus, it is important for fellowship program websites to be up to date and contain accurate and pertinent information.

Methods Twenty-nine variables, determined as important website content on the basis of prior published website analyses and from surveys of preferences, were extracted from the relevant websites of all accredited gastroenterology fellowships in the USA. Results were binary-i.e., a website either contained or did not contain each item.

Results A total of 178 websites were evaluated. The mean number of online content items was 14.1( $\pm 3.2 \mathrm{SD})$ out of a possible $29(47.1 \%)$. Program coordinator contact information, application information, and the number of current fellows were accessible on $>80 \%$ of websites. In contrast, the typical number and types of procedures performed by fellows and number of hospitals covered by fellows on call were found on $<10 \%$ of websites. Analysis revealed that $23.2 \%$ of lifestyle, $48.3 \%$ of training, and $59.6 \%$ of program variables were met.

Conclusions Gastroenterology fellowship websites lacked important content. Websites had a lower mean percentage of lifestyle content compared to training and program-related items. An organized website containing relevant information may not only attract qualified applicants but also avert unnecessary email inquiries and inappropriate applications. This study may provide guidance to gastroenterology fellowship programs seeking to improve their websites for applicants.
\end{abstract}

Keywords Gastroenterology · Fellowship · Website · Content · Comprehensiveness

Jack H. Ruddell

jack_ruddell@brown.edu

Adam E. M. Eltorai

aeltorai@gmail.com

Mena Bakhit

mena_bakhit@brown.edu

Arman M. Lateef

arman_lateef@brown.edu

Steven F. Moss

steven_moss@brown.edu

1 Warren Alpert Medical School, Brown University, Box G-A1, Providence, RI 02912, USA

2 Division of Gastroenterology and Hepatology, Department of Internal Medicine, Warren Alpert Medical School, Brown University, Providence, RI, USA

\section{Introduction}

Applying for gastroenterology fellowship is competitive; in 2018, 759 applicants applied and 489 matched, yielding a $64.4 \%$ match rate [1]. Applicants usually apply for gastroenterology fellowship approximately 1 year prior to the position start date through the Electronic Residency Application Service (ERAS) [2]. Due to the intense competition, applicants often apply to a large number of programs, many with which they are unfamiliar. Since the Internet is an essential source of information for applicants, it is important for fellowship program websites to be up to date, containing accurate and pertinent information [3-5].

While there have been prior studies of other residency and fellowship programs that have evaluated program-specific websites, none to date have examined gastroenterology fellowship websites. The purpose of this study was to assess 
gastroenterology fellowship program online content, specifically evaluating those variables that would be pertinent to prospective applicants.

\section{Materials and Methods}

Twenty-nine content variables (Table 1), derived from published website analyses of training programs in other medical disciplines [6-12] and from surveys identifying which information is most valued by applicants [4, 14], with the addition of gastroenterology-specific content, were divided into training (10 variables related to curriculum quality and depth, teaching modes, and details of schedules), program (11 variables including the program size and other general characteristics), and lifestyle (8 variables concerning remuneration, social events, call time, and the type of career path fellows followed after graduating). They were evaluated separately by two authors (JHR and AML) reviewing websites of all 189 gastroenterology fellowships accredited by the Accreditation Council for Graduate Medical Education (ACGME) as of August 2018. Discrepancies in observations were resolved by consensus. Programs without websites could not be analyzed and were excluded from the study. The number of total fellows per program and fellowship positions offered per year was collected from the ACGME's Accreditation Data System. Programs with $\geq 9$ total fellows (median) were classified as large, whereas programs with $<9$ were classified as small. The number of content items present on program websites was compared by program size and whether a program contained a 4th year advanced training position using Wilcoxon rank-sum tests. Results are expressed as mean \pm standard deviation (SD) unless otherwise stated. Results were binary, based on whether each item was included or not included in each program's publicly available website.

\section{Results}

A total of 178 publicly accessible gastroenterology fellowship websites were evaluated. Programs had a mean of $3.0 \pm 1.3$ gastroenterology fellowship positions available per year $($ median $=3)$ and a mean $9.0 \pm 4.4($ median $=9)$ total fellows per institution. There were 93 large programs $(52.2 \%)$ with $\geq 9$ fellows enrolled across years. The greatest number of programs were found in the Northeast US Census region (67 programs), compared to the fewest in the West (26 programs). Fifty-four programs $(30.3 \%)$ indicated availability of a 4th year of advanced gastroenterology training.

\section{Content Variables}

The mean number of online content items present per gastroenterology fellowship website was $13.2 \pm 4.2$ out of a possible 29 variables $(45.6 \%)$. The greatest number of items included by any fellowship website was 22 (1 program), with 8 websites containing $<5$ of the 29 variables. Hospital affiliation, program coordinator contact information, application requirements and information, and the

Table 1 Twenty-nine online content items assessed, divided into training, program, and lifestyle categories

Online content variables

\section{Training}

Away rotation information

Didactic schedule

Duration and types of rotations

Faculty members listing

Research listing/information

Program

Alumni listing

Application requirements/information

Current fellow listing

Fourth year advanced training

Hospital information

Number of current fellows

Lifestyle

Academic stipend for books, travel, research

Call roster for first years

Formal conference hours

Number of hospitals covered on call
Teaching opportunities for fellows

Typical number and types of procedures performed by fellows

Whether liver transplants are performed at the institution

Whether or not advanced endoscopic procedures are performed in training

Whether training is by hospital-employed and/or private practice physicians

Number of positions available

Program coordinator contact

Program director contact

Recent board pass rates

Synopsis/general description of the fellowship

Post-fellowship placement

Salary and benefits

Social events for trainees/social events open to the community

Typical call frequency/schedule of fellows 
number of fellows in 2018-2019 were accessible on more than $80 \%$ of program websites (Fig. 1). In contrast, only 5 of the 29 content variables were included in $<10 \%$ of program websites (Figs. 1, 2, 3): 9.6\% of programs (17/178) included conference hours; $9.0 \%(16 / 178)$ provided an example of typical numbers and types of procedures performed by fellows; $6.7 \%$ (12/178) explained whether fellows were supervised solely by hospital-employed physicians, private practice physicians, or both; $5.1 \%$ (9/178) provided board pass rates of recent graduates; and only $2.8 \%(5 / 178)$ listed the number of hospitals covered by fellows when on call. Only $23.2 \%$ of lifestyle variables $(1.9 \pm 1.3$ out of 8$)$ were listed per program website, compared with $48.3 \%$ of training $(4.8 \pm 1.8$ out of 10$)$ and $59.6 \%$ of program variables $(6.6 \pm 2.1$ out of 11$)$.

\section{Comparison of Online Content}

Large programs listed a significantly greater number of online details compared to small programs (mean 14.3 \pm 3.4 vs. $12.1 \pm 4.6$ out of 29 items; $p=0.001$ ). Programs with optional 4th year advanced gastroenterology training contained significantly more online criteria, with a mean of $15.2 \pm 3.0(52.6 \%)$ variables present, compared to $12.4 \pm 4.3$ $(42.6 \%)$ of programs without 4 th year advanced training $(p<0.001)$. fellowship websites containing each of eleven program-related online content variables
Fig. 1 Percent and number of

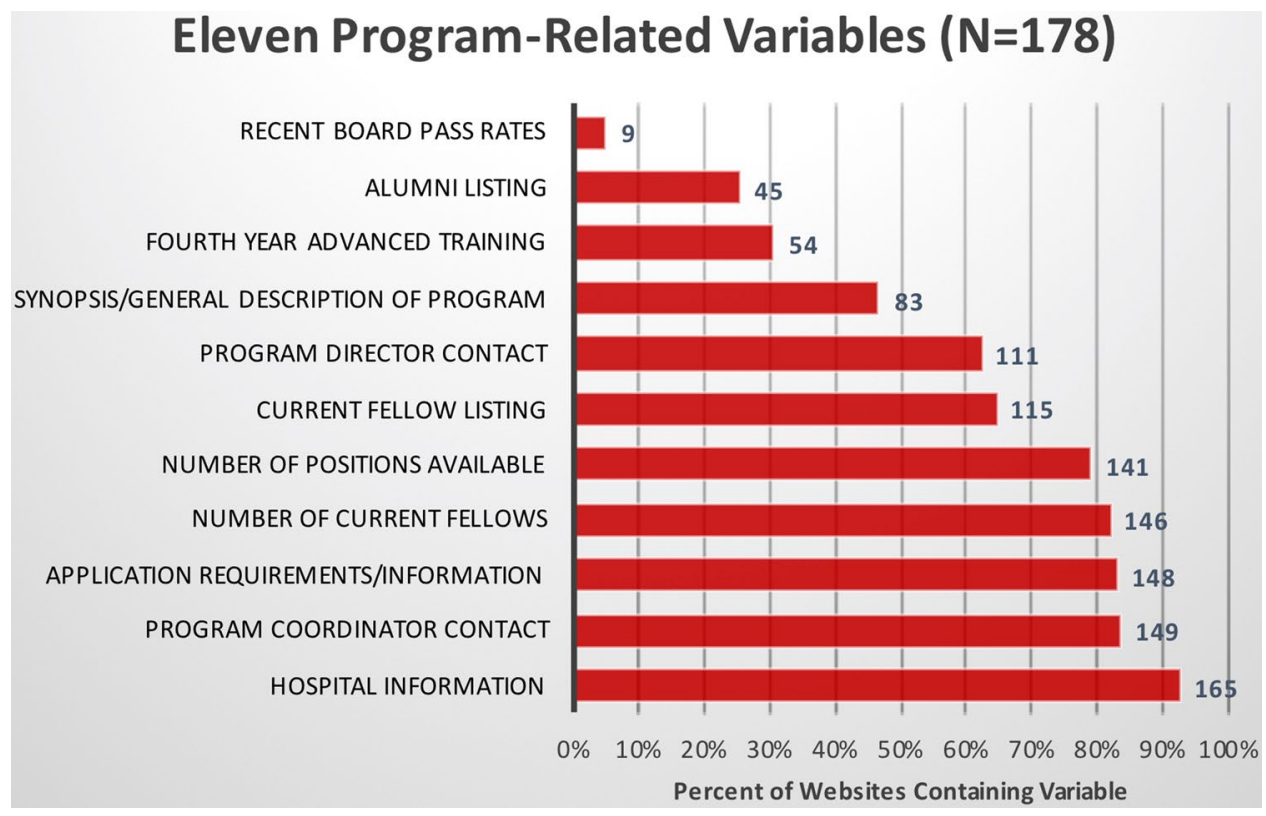

fellowship websites containing each of ten training-related online content variables

\section{Ten Training-Related Variables $(\mathrm{N}=178)$}

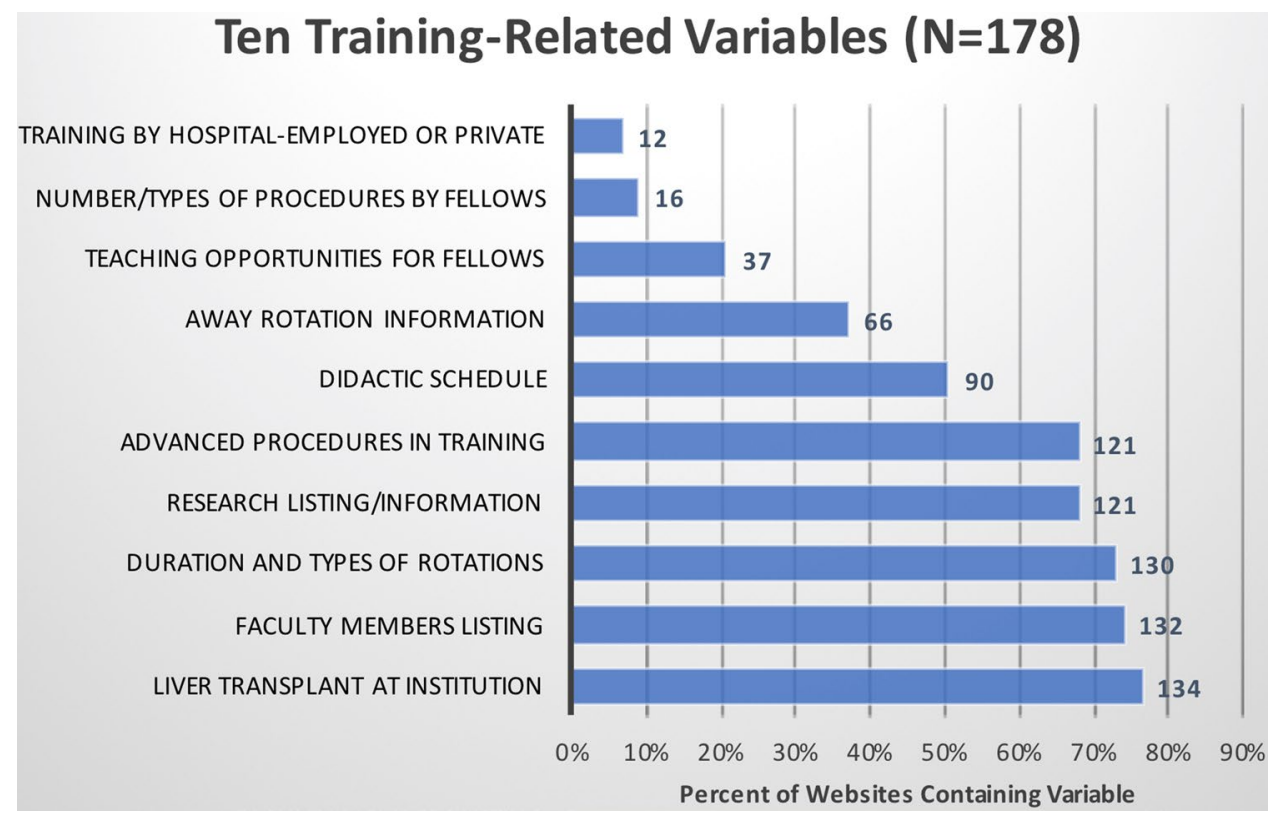


Fig. 3 Percent and number of fellowship websites containing each of eight lifestyle-related online content variables

\section{Eight Lifestyle-Related Variables ( $\mathrm{N}=178)$}

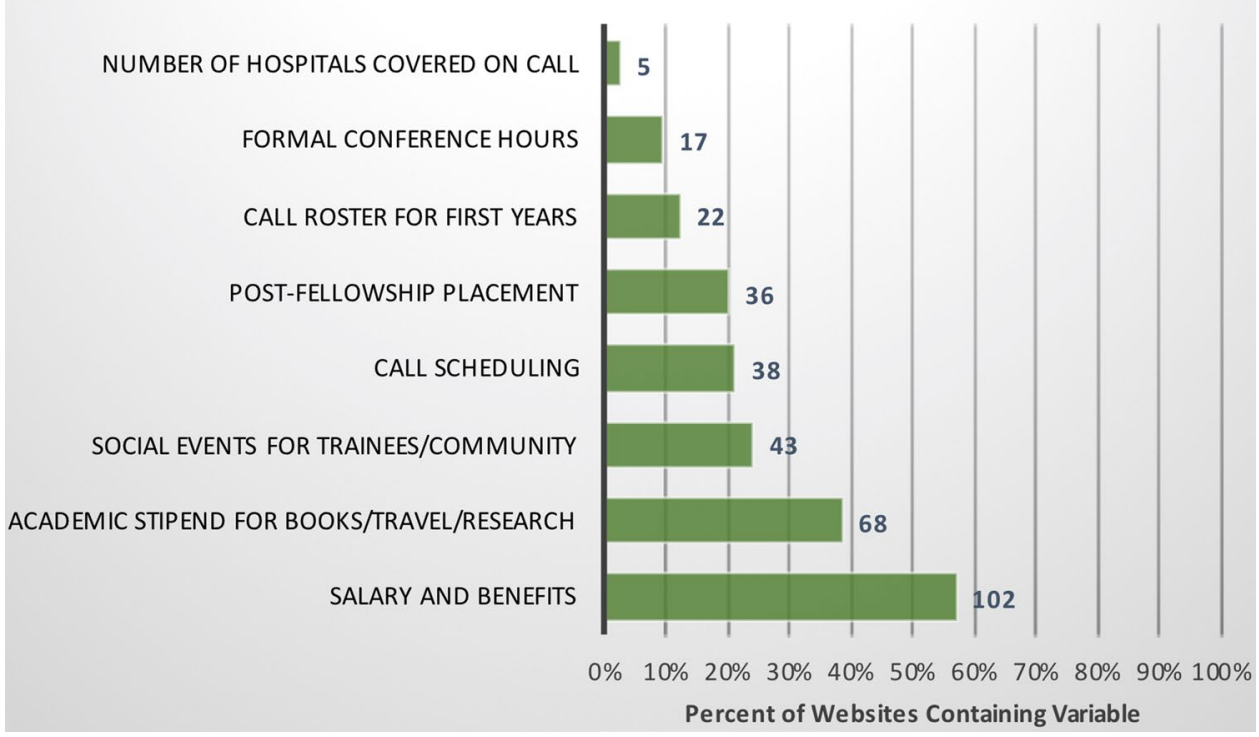

\section{Discussion}

Applicants to any specialty use the Internet extensively for program-specific information when applying for residency or fellowship training [5-10, 13]. In assessing the utility of residency and fellowship websites, several surveys of applicants have emphasized the incompleteness of pertinent information, especially concerning schedules, postresidency placement, and employment benefits [3, 4, 14]. In this study, although many websites contained information regarding hospital affiliation(s), faculty listings, program coordinator contact information, application requirements, and the total number of then-current fellows, many of the gastroenterology websites reviewed were frequently lacking in other important aspects. For example, information regarding the nature and volume of didactic teaching, typical numbers and types of procedures performed by fellows, the number of hospitals covered by the on-call fellow, the names and contact information of current fellows, the board pass rates of recently graduated fellows, and details of postfellowship placement were rarely included.

Interestingly, large programs were associated with a significantly greater number of online criteria met per program compared to small programs, possibly due to funding and personnel availability in larger programs enabling routine website maintenance. Moreover, gastroenterology programs had a significantly lower mean percentage of lifestyle content items present compared to training and program items, possibly due to a perceived lack of importance or an unwillingness to display such information.

Previous studies surveying residency applicants found that content included in a program website rather than its aesthetic qualities is of greater importance to applicants [4]. When assessing how integral websites are to applicant decision making, Chu et al. found that anesthesia residency applicants rely heavily on anesthesia program websites in order to evaluate relative program merit, prepare for interviews, and eventually formulate a rank list [14]. This was similarly noted with other specialties including emergency medicine, orthopedic surgery, otolaryngology, microsurgery, and general surgery $[5,7-10,13]$.

Limitations to this study included subjectivity in selecting items to evaluate. Many of the 29 variables were based on prior studies of other fields [6-12], as well as surveys identifying which information is most valued by applicants $[4,14]$. The variables were chosen by the authors, which included medical students, a gastroenterology fellow, and a gastroenterology attending/program director. Inclusion of a resident author, particularly one applying to gastroenterology fellowship, may have altered certain variables. Furthermore, some newly accredited programs did not have available websites and could not be included in this study. Finally, data collection occurred over several months, during which time websites may have been updated.

Gastroenterology fellowship program websites are a source of important information, providing a valuable resource for residents applying to gastroenterology fellowship. Given the Internet's omnipresence, programs may benefit from reviewing their websites through the eyes of applicants. Many gastroenterology program websites do not provide information that applicants may deem as essential. An organized website containing relevant information may not only attract applicants but also prevent unnecessary email inquiries and inappropriate applications. While 
inclusion of all 29 variables is not meant to be presented as a standard, this article may provide guidance to individual gastroenterology fellowship programs on specific areas on their websites that could be improved.

Author's contribution JHR contributed to study concept and design; acquisition of data; analysis and interpretation of data; drafting of the manuscript; critical revision of the manuscript for important intellectual content; statistical analysis. AEME contributed to study concept and design; analysis and interpretation of data; critical revision of the manuscript for important intellectual content; study supervision. MB contributed to study concept and design; analysis and interpretation of data; drafting of the manuscript; critical revision of the manuscript for important intellectual content; study supervision. AML contributed to acquisition of data; analysis and interpretation of data; critical revision of the manuscript for important intellectual content. SFM contributed to study concept and design; analysis and interpretation of data; drafting of the manuscript; critical revision of the manuscript for important intellectual content; study supervision.

\section{Compliance with ethical standards}

Conflict of interest The authors have no potential conflicts of interest to disclose.

\section{References}

1. Program TNRM. Results and Data: Specialties Matching Service 2018 Appointment Year 2018. https://mk0nrmpcikgb8jxyd19h .kinstacdn.com/wp-content/uploads/2018/02/Results-and-DataSMS-2018.pdf. Accessed 10 Oct 2018.

2. (AAMC) AoAMC. ERAS 2019 Fellowship Application Timeline 2018. https://students-residents.aamc.org/training-residency-fello wship/article/eras-fellowship-timeline/. Accessed 10 Oct 2018.

3. Embi PJ, Desai S, Cooney TG. Use and utility of Web-based residency program information: a survey of residency applicants. $J$ Med Internet Res. 2003;5:e22.
4. Gaeta TJ, Birkhahn RH, Lamont D, Banga N, Bove JJ. Aspects of residency programs' web sites important to student applicants. Acad Emerg Med. 2005;12:89-92.

5. Mahler SA, Wagner MJ, Church A, Sokolosky M, Cline DM. Importance of residency program web sites to emergency medicine applicants. J Emerg Med. 2009;36:83-88.

6. Hu J, Zhen M, Olteanu C, Avram R. An evaluation of accessibility and content of microsurgery fellowship websites. Plast Surg (Oakv). 2016;24:187-190.

7. Mulcahey MK, Gosselin MM, Fadale PD. Evaluation of the content and accessibility of web sites for accredited orthopaedic sports medicine fellowships. J Bone Joint Surg Am. 2013;95:e85.

8. Reilly EF, Leibrandt TJ, Zonno AJ, Simpson MC, Morris JB. General surgery residency program websites: usefulness and usability for resident applicants. Curr Surg. 2004;61:236-240.

9. Svider PF, Gupta A, Johnson AP, et al. Evaluation of otolaryngology residency program websites. JAMA Otolaryngol Head Neck Surg. 2014;140:956-960.

10. Silvestre J, Guzman JZ, Abbatematteo JM, Chang B, Levin LS. Evaluation of content and accessibility of hand fellowship websites. Hand (N Y). 2015;10:516-521.

11. Hansberry DR, Bornstein J, Agarwal N, McClure KE, Deshmukh SP, Long S. An assessment of radiology residency program websites. J Am Coll Radiol. 2018;15:663-666.

12. Ruddell JH, Hartley-Blossom ZJ, Bajaj AI, Grand D, Eltorai AEM. Analysis of abdominal radiology fellowship website content and comprehensiveness. Abdom Radiol (NY). 2018. https:// doi.org/10.1007/s00261-018-1861-0.

13. Yayac M, Javandal M, Mulcahey MK. Accredited orthopaedic sports medicine fellowship websites: an updated assessment of accessibility and content. Orthop J Sports Med. 2017;5:2325967116683942.

14. Chu LF, Young CA, Zamora AK, et al. Self-reported information needs of anesthesia residency applicants and analysis of applicantrelated web sites resources at 131 United States training programs. Anesth Analg. 2011;112:430-439.

Publisher's Note Springer Nature remains neutral with regard to jurisdictional claims in published maps and institutional affiliations. 